\title{
Radial Velocity Studies of Pulsations in roAp Stars: $\gamma$ Equ Revisited
}

\author{
A. Kanaan ${ }^{1}$, A. P. Hatzes ${ }^{2}$, D. Mkrtichian ${ }^{1}$
}

We have used the 2D-Coude spectrograph of the 2.7-m telescope at McDonald Observatory at a resolution of 60,000 . We use an iodine cell which provides us with permanent wavelength reference.

$\gamma$ Equ was observed for a period of approximately 6 hours. The signal to noise ratio was variable due to the presence of clouds. A typical value for the "good" spectra is 80 .

The use of a large detector provides us with complete spectral coverage from 5,000 to $6,000 \AA$ (the region where $I_{2}$ lines are most visible and useful as a wavelength reference). This allowed us to expand over our previous work analyzing the pulsations of $\gamma$ Equulei through the use of spectroscopy.

We are particularly keen in checking two conclusions from our first Paper (Kanaan \& Hatzes 1998):

- Different elements show wildly different average values of RV (radial velocity) amplitude. This conclusion has been confirmed by our reanalysis of $\gamma$ Equ, as well as our study of $33 \mathrm{Lib}$.

- There is a dependence of RV amplitude with line equivalent width. The smaller the equivalent width, the larger the RV amplitude. This is confirmed in our study of $33 \mathrm{Lib}$, but not in the new observations of $\gamma$ Equ.

With the current data we have strong indication that RV amplitudes depend on element as well as line strength. But this is not all. It is our feeling that the amplitude differences we see are predominantly caused by the non-uniform distribution of elements on the surface of these objects. To test this we are currently working on data on HR 1217 taken over half a rotation cycle (6 days) which allow us to obtain a surface mapping of all elements, as well as possible changes in amplitude ratios (between two spectral lines) with rotation phase.

\section{Reference}

Kanaan, A. \& Hatzes, A. P. 1998, ApJ, 503, 848

\footnotetext{
${ }^{1}$ Departamento de Física, Universidade Federal de Santa Catarina, Brazil

${ }^{2}$ Department of Astronomy, University of Texas at Austin, U.S.A.

${ }^{3}$ Odessa Observatory, Ukraine
} 